\title{
Oroxylin A attenuates IL-1 $\beta$-induced inflammatory reaction via inhibiting the activation of the ERK and PI3K/AKT signaling pathways in osteoarthritis chondrocytes
}

\author{
YONG ZHANG $^{1}$, QIUYAN WENG ${ }^{2}$, JIANMING CHEN ${ }^{1}$, MING LI $^{1}$ and JINMING HAN ${ }^{3}$ \\ ${ }^{1}$ Department of Trauma Orthopedics, Ningbo No. 6 Hospital; ${ }^{2}$ Department of Neurology, The Affiliated Hospital of \\ Medical School of Ningbo University; ${ }^{3}$ Department of Spine, Ningbo No. 6 Hospital, Ningbo, Zhejiang 315000, P.R. China
}

Received March 20, 2020; Accepted October 27, 2020

DOI: $10.3892 /$ etm.2021.9819

\begin{abstract}
Osteoarthritis (OA) is characterized by degradation of the articular cartilage, synovium inflammation, subchondral bone sclerosis and osteophyte formation. OA is the most common degenerative joint disorder among the elderly population. In particular, currently available therapeutic strategies, such as non-steroidal anti-inflammatory drugs (NSAIDs) may cause severe side-effects. Therefore, novel candidate targets for OA therapy are urgently needed. Oroxylin A (OrA) is a natural mono-flavonoid that can be extracted from Scutellariae radix. The present study aimed to investigate the potential effects of OrA on interleukin (IL)- $1 \beta$-induced chondrocytes inflammatory reactions. The current study performed quantitative PCR, western blotting and cell immunofluorescence to evaluate the effect of Oroxylin A in chondrocyte inflammation. The results demonstrated that OrA significantly attenuated the upregulation of inducible nitric oxide synthase and cyclooxygenase 2 by IL- $1 \beta$ at both protein and mRNA levels. IL-1 $\beta$-stimulated upregulation of matrix metalloproteinase (MMP)-3 and MMP-13 expression, in addition to disintegrin and metalloproteinase with thrombospondin motifs (ADAMTS)-4 and ADAMTS-5 expression, were all inhibited by OrA. Treatment with OrA significantly reversed the degradation of type II collagen and aggrecan by IL-1 $\beta$. Mechanistically, OrA suppressed the IL- $1 \beta$ induced activation of ERK $1 / 2$ and PI3K/AKT signaling pathways. In conclusion, these findings suggest that OrA can serve as a potential therapeutic agent for the treatment of OA.
\end{abstract}

Correspondence to: Professor Jinming Han, Department of Spine, Ningbo No. 6 Hospital, 1059 Zhongshan East Road, Ningbo, Zhejiang 315000, P.R. China

E-mail: hanjinming83@163.com

Key words: Oroxylin A, interleukin-1 $\beta$, osteoarthritis, extracellular signal-regulated kinase 1/2, phosphatidylinositol 3-kinase/protein kinase B

\section{Introduction}

Osteoarthritis (OA) is the most common degenerative joint disorder, which leads to chronic bone and muscle pain (1). OA is characterized by degradation of articular cartilage, synovial inflammation, subchondral bone sclerosis and osteophyte formation $(2,3)$. Current strategies for treating OA remain limited and did not seem to improve the management of OA. To date, application of nonsteroidal anti-inflammatory drugs (NSAIDs) is the primary therapeutic approach for the treatment of OA (4). However, side-effects, including peptic ulcers, hemorrhage and perforations, frequently occur during the therapeutic process $(5,6)$. Therefore, the development of novel agents for treating OA remain urgently sort after.

OA exhibits several risk factors (7), where the inflammatory mediator interleukin (IL)- $1 \beta$ has been shown to serve an important role in its pathogenesis (8). The adult articular cartilage consists of the extracellular matrix (ECM) and chondrocytes (9). ECM provides the necessary tension and strength to the articular cartilage (10). Under physiological conditions, a subtle balance between ECM synthesis and degradation maintains the homeostasis of the cartilage (11). Previous studies have suggested that IL-1 $\beta$ significantly stimulates chondrocytes to secrete matrix metalloproteinases (MMPs) $(12,13)$ and promotes the production of inflammatory mediators, including prostaglandin E2 and nitric oxide (NO) (14). It has been suggested that MMPs are responsible for the degradation of ECM during the progression of OA (15). ECM is composed of type II collagen, proteoglycans and aggrecan $(16,17)$. In previous studies, IL-1 $\beta$ has been reported to downregulate the expression of type II collagen and aggrecan in vitro, thereby leading to the degradation of articular cartilage $(18,19)$. Therefore, agents targeting the IL-1 $\beta$-induced inflammatory response during the pathogenesis of OA can potentially attenuate the progression of OA.

Oroxylin A (OrA) is a natural mono-flavonoid that can be extracted from the herb Scutellariae radix (20). Accumulating evidence has demonstrated that OrA exerts multiple pharmacological effects, including anti-inflammatory $(21,22)$, anti-oxidative $(23,24)$ and anti-tumorigenic $(25,26)$ properties. Therefore, it has been extensively used to treat a variety of diseases. The anti-inflammatory effects of OrA are mainly 
mediated by blocking the phosphorylation and subsequent activation of the PI3K/AKT signaling pathway (27). By contrast, a previous study has also reported that OrA reduces lipopolysaccharide-induced inflammatory reactions by activating the $\mathrm{NF}-\kappa \mathrm{B}$ signaling pathway (28). However, the comprehensive role of OrA in OA progression remains poorly understood.

The present study aimed to perform western blotting, qPCR and cell immunofluorescence to evaluate the protective effect of OrA on IL-1 $\beta$-induced chondrocyte inflammation and its underlying mechanism.

\section{Materials and methods}

Reagents. OrA and the Cell Counting Kit (CCK)- 8 were purchased from MedChemExpress. Primary antibodies against the unphosphorylated forms of PI3K (cat. no. 4255), AKT (cat. no. 9272), ERK (cat. no. 4695), p38 (cat. no. 14451), JNK (cat. no. 9252), p65 (cat. no. 8242), NF- $\kappa \mathrm{B}$ inhibitor $\alpha$ (I $\mathrm{B} \alpha$; cat. no. 4814), inducible nitric oxide synthase (iNOS; cat. no. 39898), cyclooxygenase 2 (COX-2; cat. no. 12282) and $\beta$-actin (cat. no. 3700), and the phosphorylated (p) forms of AKT (cat. no. 13038), ERK (cat. no. 4370), p38 (cat. no. 9216), JNK (cat. no. 9251), and p65 (cat. no. 3039) were obtained from Cell Signaling Technology, Inc. Primary antibodies against MMP3 (cat. no. 17873-1-AP) and MMP13 (cat. no. 18165-1-AP) were purchased from Proteintech Group, Inc., whereas those against aggrecan, disintegrin and metalloproteinase with thrombospondin motifs ADAMTS-4 (cat. no. ab185722), ADAMTS-5 (cat. no. ab41037) and type II collagen (cat. no. ab188570) were from purchased from Abcam. Secondary antibodies (anti-mouse cat. no. 7076 and anti-rabbit cat. no. 7074) were obtained from Cell Signaling Technology, Inc and diluted in secondary antibody diluent (Beyotime Institute of Biotechnology; cat. no. P0258; 1:100). DMEM/Ham's F12 medium (DMEM/F12) was obtained from Hyclone, Cytiva. FBS was purchased from Gibco (Thermo Fisher Scientific, Inc.) and recombinant rat IL-1 $\beta$ (cat. no. 211-11B) was purchased from PeproTech, Inc.

Isolation and culture of primary chondrocytes. A total of 30 C57BL/6 mice (age, 2-3 days) were purchased from the Animal Center of Chinese Academy of Sciences and were decapitated before chondrocytes were isolated from their articular cartilage. Briefly, the articular cartilages of each mouse were carefully extracted under aseptic conditions and cut into $\sim 1-2 \mathrm{~mm}^{2}$ slices, followed by washing with PBS three times at room temperature. The pieces were then digested using with DMEM/F12 medium supplemented with $0.1 \%$ collagenase II at $37^{\circ} \mathrm{C}$ in a humidified atmosphere containing $5 \% \mathrm{CO}_{2}$ for $8 \mathrm{~h}$. The cells were then collected via centrifugation at $1,000 \mathrm{x}$ g for $3 \mathrm{~min}$ at $25^{\circ} \mathrm{C}$, washed with PBS three times, plated into cell culture flasks in DMEM/F12 supplemented with $10 \% \mathrm{FBS}, 100 \mathrm{U} / \mathrm{ml}$ penicillin and $100 \mathrm{mg} / \mathrm{ml}$ streptomycin and incubated at $37^{\circ} \mathrm{C}$ in an atmosphere of $95 \%$ air and $5 \% \mathrm{CO}_{2}$. The medium was changed after $24 \mathrm{~h}$ and the cells were harvested when $80-90 \%$ confluence was reached. Only chondrocytes from passages 1-2 were used in the present study to avoid the loss of phenotype. Light microscopy (upper panel original magnification, $\mathrm{x} 100$; lower panel original magnification, $\mathrm{x} 200$ ) was performed to observe the cell morphology of chondrocytes. Cells at passages 1-2 had a rounded or polygonal structure.
$C C K-8$ assay. The primary chondrocytes were plated at a density of $8 \times 10^{3}$ cells/well into 96 -well plates followed by treatment with DMEM/F12 medium containing increasing concentrations of $\operatorname{OrA}(0,2,4,8,16,32,64$ and $128 \mu \mathrm{M})$ at $37^{\circ} \mathrm{C}$ in a humidified atmosphere containing $5 \% \mathrm{CO}_{2}$ for 24 and $48 \mathrm{~h}$. At the end of each time point, $10 \mu \mathrm{l} \mathrm{CCK}-8$ solution was added into each well and the chondrocytes were incubated further for an additional $4 \mathrm{~h}$ at $37^{\circ} \mathrm{C}$ in an atmosphere with $95 \%$ air and $5 \% \mathrm{CO}_{2}$. Absorbance was then measured at $450 \mathrm{~nm}$ using a Multiskan ${ }^{\mathrm{TM}} \mathrm{GO}$ microplate reader (Thermo Fisher Scientific, Inc.).

$R N A$ extraction and reverse transcription-quantitative $P C R$ $(R T-q P C R)$. The primary chondrocytes were seeded into six-well plates at a density of $5 \times 10^{5}$ cells/well. Once they adhered to the plates, chondrocytes were pre-treated with various concentrations of OrA $(4,8$, and $16 \mu \mathrm{M})$ for $2 \mathrm{~h}$ before being stimulated with or without IL-1 $\beta(10 \mathrm{ng} / \mathrm{ml})$ at $37^{\circ} \mathrm{C}$ in a $5 \% \mathrm{CO}_{2}$ incubator for $24 \mathrm{~h}$. Total RNA was extracted using a TRIzol ${ }^{\circledR}$ reagent (Thermo Fisher Scientific, Inc.) according to the manufacturer's protocol. Subsequently, a total of 1,000 ng RNA was reverse transcribed into cDNA (PrimeScript ${ }^{\mathrm{TM}}$ Reverse Transcriptase kit; Takara Biotechnology Co., Ltd.) using the following temperature protocol: $30^{\circ} \mathrm{C}$ for $10 \mathrm{~min}$, $42^{\circ} \mathrm{C}$ for $30 \mathrm{~min}$ and $70^{\circ} \mathrm{C}$ for $15 \mathrm{~min}$, after which the sample was cooled on ice.

RT-qPCR was conducted using the SYBR green Master Mix (Takara Biotechnology Co., Ltd.) and performed with the ViiA ${ }^{\mathrm{TM}} 7$ real-time PCR system (Thermo Fisher Scientific, Inc.) according to the manufacturer's protocol. The thermocycling conditions of qPCR were as follows: $95^{\circ} \mathrm{C}$ for $10 \mathrm{~min}$, followed by 50 cycles of $95^{\circ} \mathrm{C}$ for $15 \mathrm{sec}$ and $60^{\circ} \mathrm{C}$ for $1 \mathrm{~min}$. The cycle threshold $\left(C_{t}\right)$ of each sample was normalized to the expression levels of $\beta$-actin. The $2^{-\Delta \Delta \mathrm{Cq}}$ method was used to assess the relative expression of various target genes (29). The primer sequences of tumor necrosis factor- $\alpha$ (TNF- $\alpha$ ), IL-6, iNOS, MMP-3, MMP-13 and $\beta$-actin are listed in Table I.

Immunofluorescence microscopy. Primary chondrocytes $\left(5 \times 10^{4}\right.$ cells $\left./ \mathrm{ml}\right)$ were seeded into a 12 -well plate and were then stimulated at $37^{\circ} \mathrm{C}$ in a humidified atmosphere containing $5 \% \mathrm{CO}_{2}$ for $48 \mathrm{~h}$. Cells were fixed with $4 \%$ paraformaldehyde for $30 \mathrm{~min}$ at $25^{\circ} \mathrm{C}$ and washed with PBS. Subsequently, the cell slides were treated with $0.1 \%$ Triton X-100 for $10 \mathrm{~min}$ at room temperature, blocked with $10 \%$ bovine serum albumin for $1 \mathrm{~h}$ at $25^{\circ} \mathrm{C}$ and then incubated with primary antibodies against COX-2 (dilution, 1:500), MMP3 (dilution, 1:500), MMP13 (dilution, 1:500) or type II collagen (dilution, 1:200) at $4^{\circ} \mathrm{C}$ overnight. The following day, cells were incubated with fluorescein-conjugated goat anti-rabbit IgG antibody (Abcam; cat. nos. ab150077 and ab150115) diluted in immunofluorescence secondary antibody diluent (Beyotime Institute of Biotechnology; cat. no. P0265; 1:100) for $1 \mathrm{~h}$ at room temperature in the dark. Subsequently, the cell nuclei were treated with $0.05 \%$ DAPI (Beyotime Institute of Biotechnology; cat. no. C1002) for an additional $5 \mathrm{~min}$ at room temperature in the dark. All images were captured using a fluorescence microscope (Olympus Corporation). Fluorescence intensity was measured using ImageJ software (v. d1.47; National Institutes of Health). 
Table I. Sequences of primers used in reverse transcription-quantitative PCR.

\begin{tabular}{lll}
\hline Gene & \multicolumn{1}{c}{ Forward primer } & \multicolumn{1}{c}{ Reverse primer } \\
\hline TNF- $\alpha$ & 5'-GGAACACGTCGTGGGATAATG-3' & 5'-GGCAGACTTTGGATGCTTCTT-3' \\
IL-6 & 5'-GGCGGATCGGATGTTGTGAT-3' & 5'-GGACCCCAGACAATCGGTTG-3' \\
iNOS & 5'-CAGGGAGAACAGTACATGAACAC3' & 5'-TTGGATACACTGCTACAGGGA-3' \\
MMP-3 & 5'-TTAAAGACAGGCACTTTTGGCG-3' & 5'-CCCTCGTATAGCCCAGAACT-3' \\
MMP-13 & 5'-CTATCCCTTGATGCCATTACCAG-3' & 5'-ATCCACATGGTTGGGAAGTTC-3' \\
$\beta$-actin & 5'-AGCCATGTACGTAGCCATCC-3' & 5'-CTCTCAGCAGTGGTGGTGAA-3'
\end{tabular}

TNF- $\alpha$, tumor necrosis factor- $\alpha$; IL, interleukin; MMP, matrix metalloproteinase; iNOS, inducible nitric oxide synthase.

Western blot analysis. To measure the expression levels of iNOS, COX-2, MMP-3, MMP-13, aggrecan, ADAMTS-4, ADAMTS-5 and type II collagen, a total of $5 \times 10^{5}$ chondrocytes/well were seeded into six-well plates and pre-treated with various concentrations of $\operatorname{OrA}(4,8$ and $16 \mu \mathrm{M})$ for $2 \mathrm{~h}$, followed by stimulation with or without IL-1 $\beta(10 \mathrm{ng} / \mathrm{ml})$ at $37^{\circ} \mathrm{C}$ in a $5 \% \mathrm{CO}_{2}$ incubator for $24 \mathrm{~h}$. To explore the molecular mechanism of OrA in the progression of OA, a total of $5 \times 10^{5}$ chondrocytes/well were seeded into six-well plates, pre-treated with or without $16 \mu \mathrm{M}$ OrA for $2 \mathrm{~h}$ and then stimulated with or without IL-1 $\beta(10 \mathrm{ng} / \mathrm{ml})$ for different time periods $(0,15,30$ and $60 \mathrm{~min})$ at $37^{\circ} \mathrm{C}$ in a $5 \% \mathrm{CO}_{2}$ incubator. Following treatment, total proteins were extracted using a RIPA lysis buffer containing $1 \%$ protease and phosphorylase inhibitors (Beyotime Institute of Biotechnology). Proteins were then incubated on ice for an additional $30 \mathrm{~min}$ and centrifuged at $12,000 \times \mathrm{g}$ for $10 \mathrm{~min}$ at $4^{\circ} \mathrm{C}$. Total protein concentration was measured using a bicinchoninic acid protein assay kit (Beyotime Institute of Biotechnology). A total of $40 \mu \mathrm{g}$ of protein from each group was separated using SDS-PAGE on a $10 \%$ gel and then transferred onto $0.22-\mu \mathrm{m}$ PVDF membranes. Following blocking with 5\% non-fat dry milk for $2 \mathrm{~h}$ at room temperature, membranes were incubated with primary antibodies against iNOS, COX-2, MMP3, MMP-13, ADAMTS-4, ADAMTS-5, PI3K, p-AKT, AKT, p-ERK, ERK, p-p38, p38, p-JNK, JNK, p-p65, p65, IкB $\alpha, \beta$-actin (all in dilution 1:1,000) or type II collagen (dilution, 1:500) overnight at $4^{\circ} \mathrm{C}$. The membranes were then washed three times with TBS- $0.1 \%$ Tween-20 for 5 min each time. Subsequently, the membranes were incubated with the secondary antibodies (Cell Signaling Technology, Inc.; anti-mouse cat. no. 7076 and anti-rabbit cat. no. 7074) diluted in secondary antibody diluent (Beyotime Institute of Biotechnology; cat. no. P0258; 1:100) for $2 \mathrm{~h}$ at room temperature. The protein bands were visualized by using electrochemiluminescence (Beyotime Institute of Biotechnology; cat. no. P0018FS) and captured by a BioSpectrum imaging system (Thermo Fisher Scientific, Inc.) and densitometry analysis was performed using the ImageJ software (v. d1.47; National Institutes of Health).

Animals. Animal experiments were conducted in accordance with the International Ethical guidelines (30) and the National Institutes of Health Guide for Care and Use of Laboratory Animals (NIH Pub No 85-23, revised 1996) (31). The procedures were approved by the Ethics Committee of Ningbo No. 6 Hospital (Ningbo, China).
Statistical analysis. Data are expressed as the mean \pm standard deviation (SD) from three experimental repeats. The GraphPad Prism software (version 7.0; GraphPad Software Inc.) was applied for all statistical analyses. One-way ANOVA followed by Tukey's post hoc test was performed to detect significant differences among groups. $\mathrm{P}<0.05$ was considered to indicate a statistically significant difference.

\section{Results}

Effects of OrA on murine chondrocyte viability. Chondrocytes were extracted from the articular cartilage of each mice and their morphology was examined. The results revealed that chondrocytes in the cartilage matrix had a rounded or polygonal structure (Fig. 1A). Chondrocytes were seeded into 96-well plates at a density of $8 \times 10^{3}$ cells/well. After adhesion to the dishes, cells were treated with ascending concentrations of OrA $(0,2,4,8,16,32,64$, and $128 \mu \mathrm{M})$ for 24 and $48 \mathrm{~h}$. The results revealed that OrA at lower concentrations $(0,2,4,8$, and $16 \mu \mathrm{M})$ was not toxic for chondrocytes (Fig. 1B), while the higher concentrations $(>32 \mu \mathrm{M})$ were toxic for chondrocytes, with the majority of cells dying after stimulation.

OrA attenuates $I L-1 \beta$-induced inflammation. The potentially protective effects of OrA against IL-1 $\beta$-induced inflammatory reaction was next determined by RT-qPCR and western blotting. Although IL-1 $\beta$ significantly promoted the expression of the inflammatory factors TNF- $\alpha$, IL- 6 and iNOS, treatment with OrA significantly reversed this effect in a dose-dependent manner (Fig. 2A). Furthermore, western blot analysis revealed that $16 \mu \mathrm{M}$ OrA markedly inhibited IL-1 $\beta$-induced upregulation of iNOS and COX-2 (Fig. 2B and C). The protective effects of OrA on IL-1 $\beta$-induced inflammatory reaction was also supported by results from immunofluorescence analysis. Significantly lower expression levels of COX-2 were observed in cells pre-treated with OrA compared with those in cells treated with IL-1 $\beta$ alone without OrA pre-treatment (Fig. 2D and E).

OrA reverses $I L-1 \beta$-induced degradation of ECM. To explore the effect of OrA on IL-1 $\beta$-induced degradation of ECM, RT-qPCR was first performed to evaluate the expression levels of the matrix-degrading enzymes MMP-3 and MMP-13. The results demonstrated that OrA significantly attenuated the IL-1 $\beta$-mediated upregulation of MMP-3 and MMP-13 mRNA (Fig. 3A). The $16 \mu \mathrm{M}$ OrA-mediated protective effects 

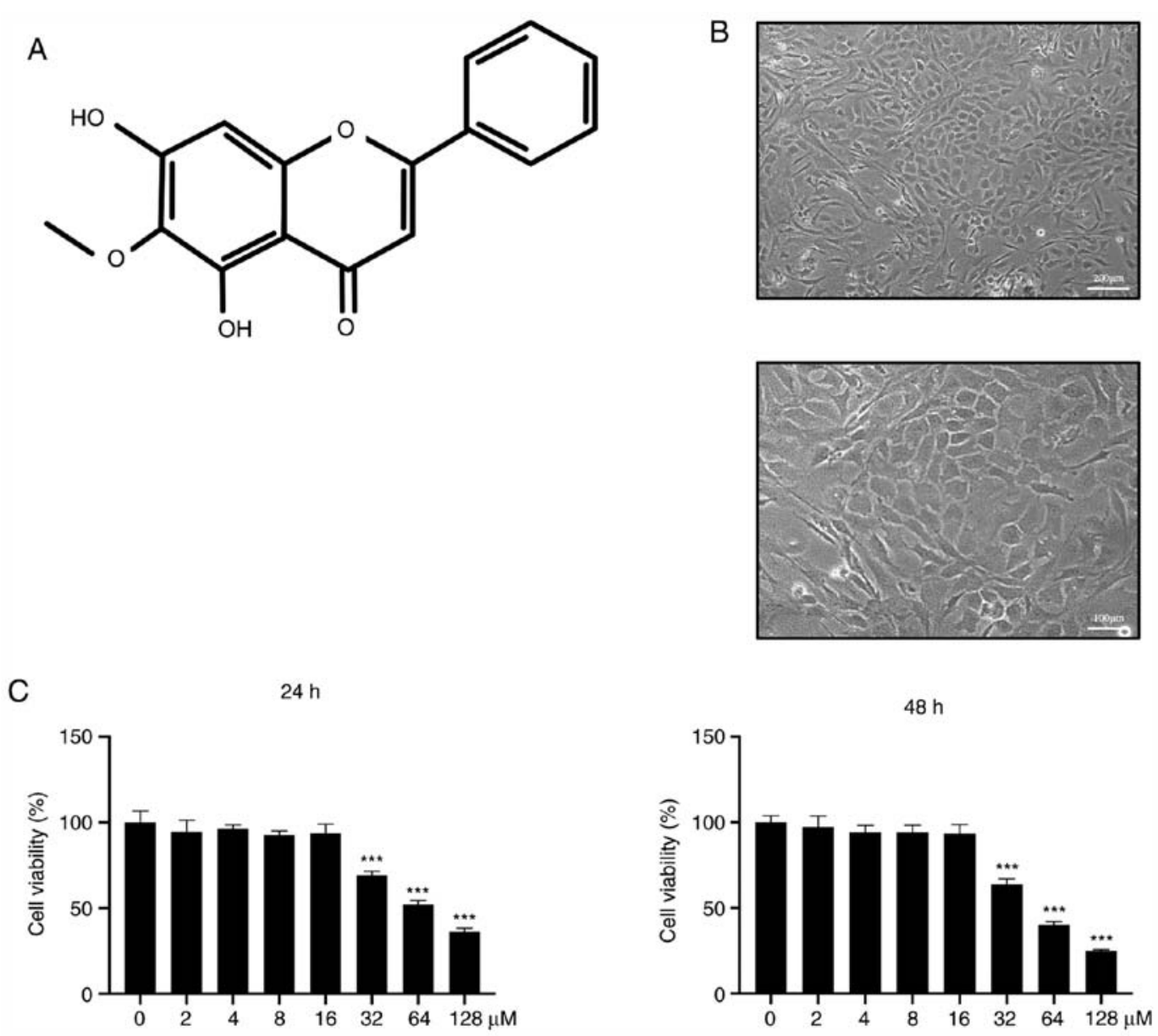

Figure 1. Effect of OrA on murine chondrocyte viability. (A) Chemical structure of OrA. (B) Morphology of chondrocytes (upper panel original magnification, x 100; lower panel original magnification, $\mathrm{x} 200)$. (C) Chondrocytes were treated with medium supplemented with different concentrations of OrA $(0,2,4$, $8,16,32,64$ and $128 \mu \mathrm{M}$ ) for 24 and $48 \mathrm{~h}$ before the CCK-8 assay was performed to assess cell viability. Each experiment was repeated three times independently. Data are expressed as the mean \pm standard deviation. ${ }^{* * *} \mathrm{P}<0.001$ vs. untreated. OrA, oroxylin A; CCK-8, Cell Counting Kit.

against IL-1 $\beta$-induced MMP-3 and MMP-13 upregulation was also confirmed on protein level using western blot analysis (Fig. 3B and C). Consistent with the previous findings of the present study, immunofluorescence results supported the potentially suppressive effects of OrA on IL-1 $\beta$-mediated increased expression of MMP-3 and MMP-13. Significantly lower expression levels of MMP-3 and MMP-13 were observed in cells pre-treated with OrA compared with those in cells treated with IL-1 $\beta$ alone without OrA pre-treatment (Fig. 3D and E).

The ADAMTS family of metalloproteases has been previously reported to be involved in the cleavage of aggrecan (32). Therefore, the present study investigated the effects of OrA on IL-1 $\beta$-induced expression of ADAMTS- 4 and ADAMTS-5. The results revealed that pre-treatment with $16 \mu \mathrm{M}$ OrA markedly attenuated IL-1 $\beta$-mediated upregulation of ADAMTS-4 and ADAMTS-5 (Fig. 3B and C).

Type II collagen and aggrecan are the main components of ECM $(33,34)$. Treatment with IL-1 $\beta$ significantly decreased the expression of both molecules, whilst pre-treatment with 8 and $16 \mu \mathrm{M}$ OrA significantly prevented this effect (Fig. 4A and B). Immunofluorescence analysis of type II collagen also indicated that OrA pre-treatment effectively protected against IL-1 $\beta$-mediated downregulation of type II collagen (Fig. 4C and D).
Effect of OrA on IL-1 $\beta$-induced $N F-\kappa B$ and MAPK activation. Western blot analysis indicated that cell stimulation with IL-1 $\beta$ for 15 min markedly increased the phosphorylation of p65 and I $\mathrm{B} \alpha$ degradation (Fig. 5A and B). However, pre-treatment with OrA had no effects on the NF- $\mathrm{KB}$ signaling pathway activation (Fig. 5A and B). The effect of OrA on IL-1 $\beta$-mediated phosphorylation of ERK, JNK and $\mathrm{p} 38$ was subsequently investigated using western blot analysis. Although OrA exerted no effects on the activation of JNK and p38, it significantly prevented the IL-1 $\beta$-mediated phosphorylation of ERK at 15 min compared with that cells that were not pre-treated with OrA (Fig. 5C and D).

Effect of OrA on IL-1 $\beta$-mediated PI3K/AKT activation. To further explore the potential anti-inflammatory effects of OrA in chondrocytes, western blot analysis was applied to evaluate the phosphorylation levels of AKT. The results demonstrated that at 15 and $30 \mathrm{~min}$, IL-1 $\beta$-mediated AKT phosphorylation was significantly lower in chondrocytes pre-treated with OrA compared with cells there were not pre-treated (Fig. 6A and B).

\section{Discussion}

$\mathrm{OA}$ is a chronic age-associated degenerative joint disease with a complex pathology that imposes severe socio-economic 


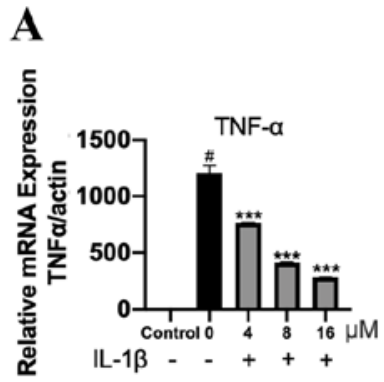

B

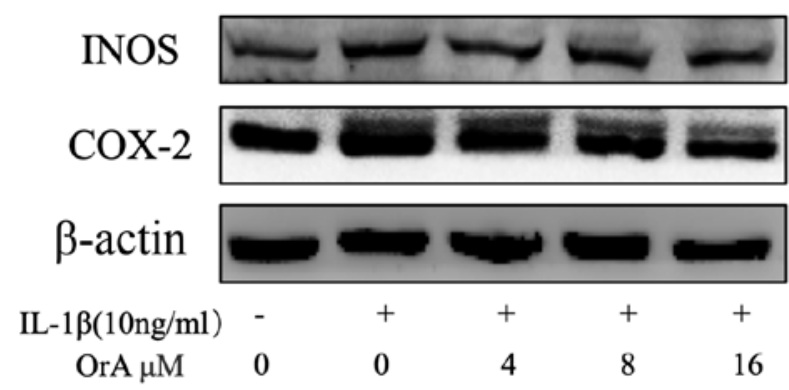

D

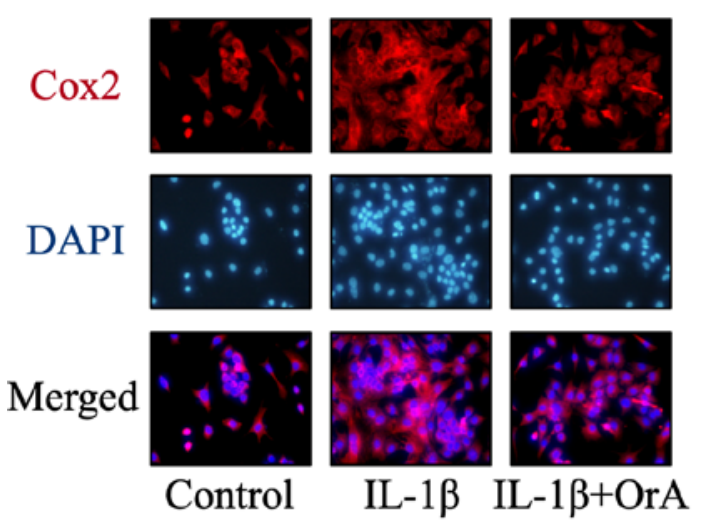

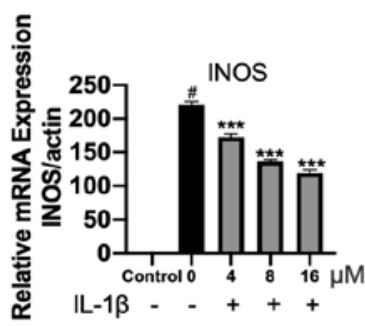

C
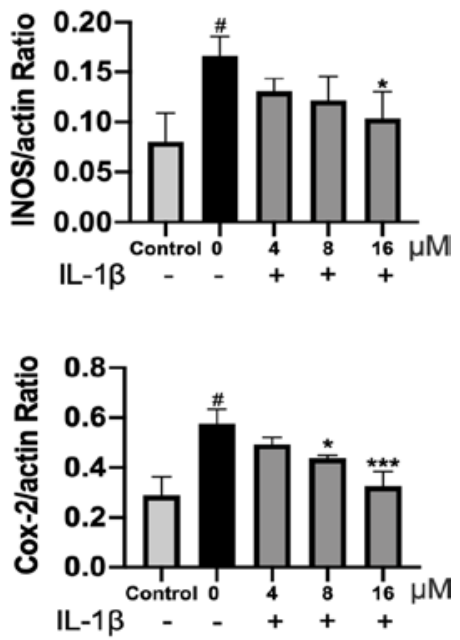

E

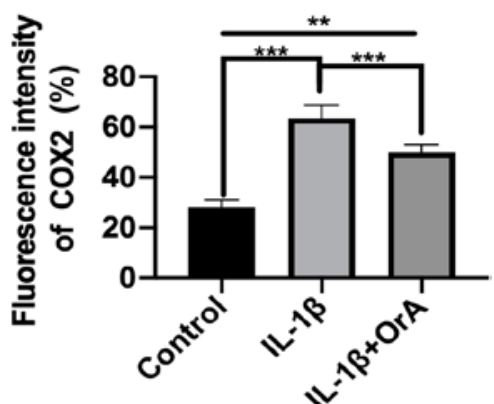

Figure 2. OrA attenuates IL-1 $\beta$-induced inflammation. Chondrocytes were treated with various concentrations of OrA $(4,8$, and $16 \mu \mathrm{M})$ and stimulated with or without IL-1 $(10 \mathrm{ng} / \mathrm{ml})$ for $24 \mathrm{~h}$. (A) Relative mRNA expression levels of TNF- $\alpha$, IL- 6 and iNOS were determined by reverse-transcription-quantitative PCR. ${ }^{*} \mathrm{P}<0.01$ vs. untreated; ${ }^{* *} \mathrm{P}<0.01$ and ${ }^{* * *} \mathrm{P}<0.001$ vs. IL-1 $\beta$ only. (B) Protein expression levels of iNOS and COX-2 were determined by western blot analysis. (C) Quantification of iNOS and COX-2 expression. ${ }^{\#} \mathrm{P}<0.01$ vs. untreated; ${ }^{*} \mathrm{P}<0.05$ and ${ }^{* * *} \mathrm{P}<0.001$ vs. IL-1 $\beta$ only. (D) Immunofluorescence analysis of COX-2 expression, (E) which was quantified. Original magnification, x200. Each experiment was repeated three times independently. Data are expressed as the mean \pm standard deviation. ${ }^{* *} \mathrm{P}<0.01$ and ${ }^{* * *} \mathrm{P}<0.001$. OrA, oroxylin A; IL-1 $\beta$, interleukin-1 $\beta$; TNF- $\alpha$, tumor necrosis factor $\alpha$; iNOS, inducible nitric oxide synthase; COX-2, cyclooxygenase 2 .

burdens on the patients (35). According to statistics, $10 \%$ men and $13 \%$ women aged $\geq 60$ years are afflicted with knee OA in the USA (36). At present, OA treatment strategies for relieving the pain symptoms are limited, where surgery is considered to be the final option in cases of advanced disease progression (37). Although agents are available to clinically relieve pain, severe side effects frequently occur. For instance, NSAIDs, which are used widely in OA to clinically relieve pain and swelling, do not ameliorate cartilage degeneration and are associated with gastrointestinal side effects, such as gastrorrhagia $(38,39)$. Therefore, novel, safe and effective alternative strategies are urgently sorted for OA treatment. OrA is a natural mono-flavonoid that can be extracted from
Scutellariae radix (40). Previous studies have reported the anti-inflammatory effects of OrA $(21,41)$. The present study investigated the potential effects of OrA in IL-1 $\beta$-induced inflammation in murine chondrocytes. The results revealed that OrA pre-treatment resulted in the suppression of inflammation by inhibiting the ERK and PI3K/AKT signaling pathways.

Accumulating evidence has indicated that OA is characterized by cartilage degeneration (42). Under normal conditions, the joint cartilage is maintained through a delicate balance between the synthesis and degradation of ECM (43). However, inflammatory cytokines, especially IL-1 $\beta$, can perturb this balance, which leads to cartilage degradation (44). In the 
A

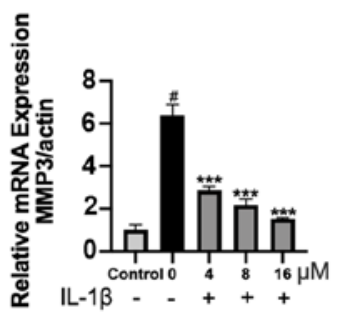

C
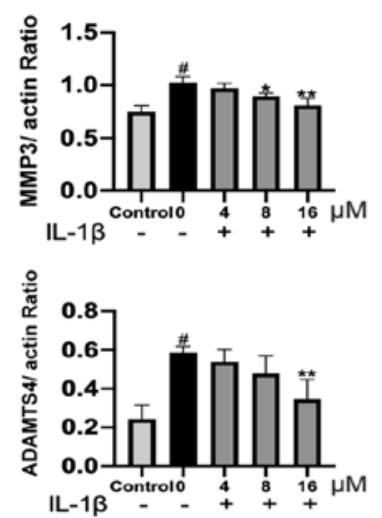
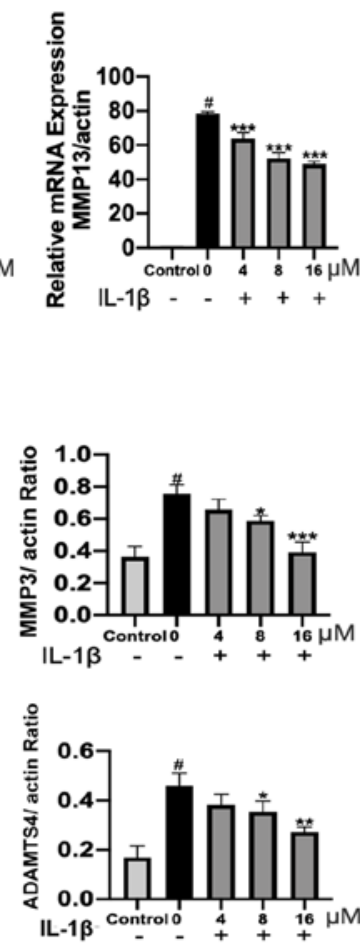

ADAMTS5

B

MMP-3

MMP-13

ADAMTS4
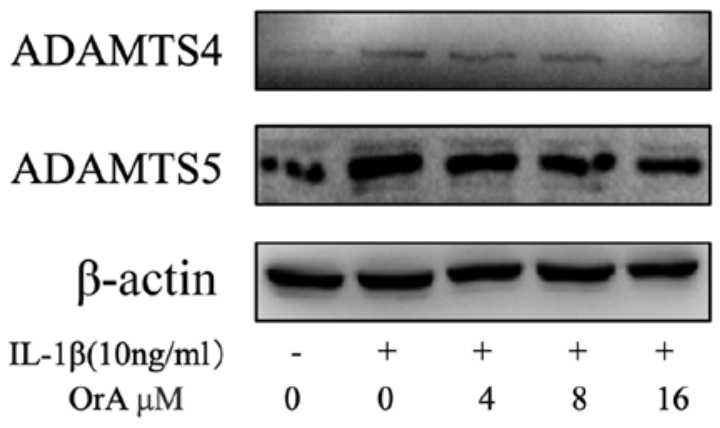
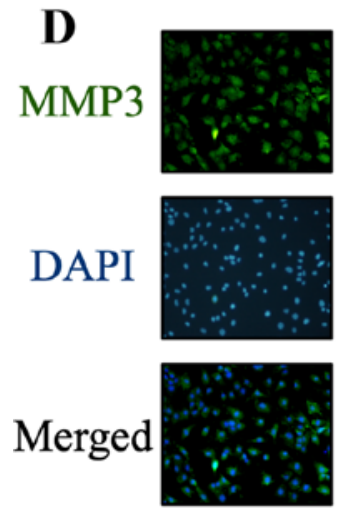

Control
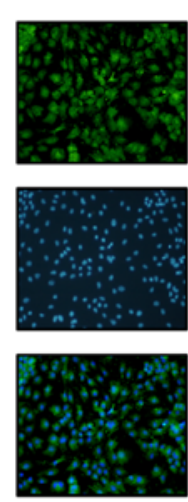

IL-1 $\beta$

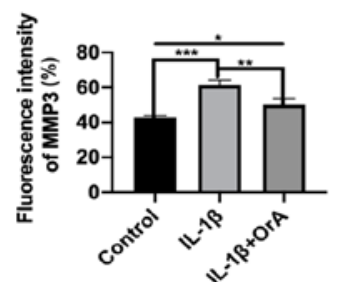

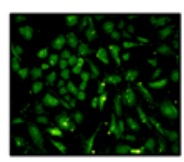
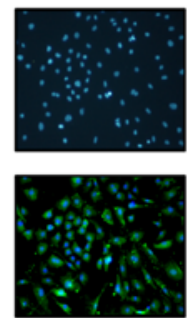

IL- $1 \beta+$ OrA
EMP13
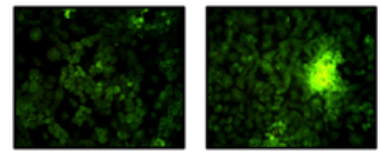

DAPI
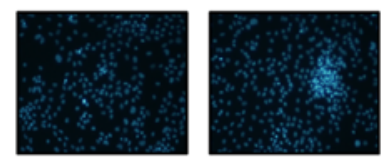

Merged

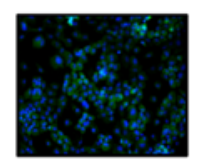

Control

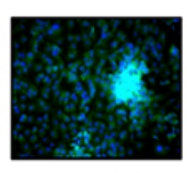

IL-1 $\beta$ IL-1 $\beta+$ OrA

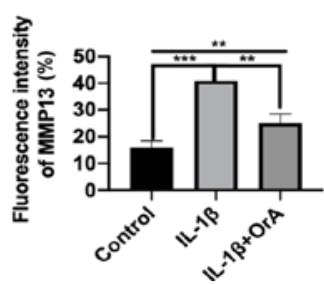

Figure 3. OrA treatment prevents IL-1 $\beta$-induced degradation of ECM. Chondrocytes were treated with various concentrations of OrA (4, 8 and $16 \mu \mathrm{M})$ and stimulated with or without IL-1 $\beta$ (10 ng/ml) for $24 \mathrm{~h}$. (A) Relative mRNA expression levels of MMP-3 and MMP-13 were determined by reverse transcrtiption-quantitative PCR. (B) The protein expression levels of MMP-3, MMP-13, ADAMTS-4 and ADAMTS-5 were determined by western blot analysis. (C) Quantification of MMP-3, MMP-13, ADAMTS-4 and ADAMTS-5 expression. Data are expressed as the mean \pm standard deviation from three experimental repeats. ${ }^{\#} \mathrm{P}<0.01$ vs. untreated; ${ }^{*} \mathrm{P}<0.05,{ }^{* *} \mathrm{P}<0.01$ and ${ }^{* * * *} \mathrm{P}<0.001$ vs. IL-1 $\beta$ only. Immunofluorescence analysis of (D) MMP-3 and (E) MMP-13 expression, which were quantified (original magnification, x100). Data are expressed as the mean \pm standard deviation from three experimental repeats. ${ }^{*} \mathrm{P}<0.05,{ }^{* *} \mathrm{P}<0.01$ and ${ }^{* * *} \mathrm{P}<0.001$. OrA, oroxylin A; ECM, extracellular matrix; IL-1 $\beta$, interleukin-1 $\beta$; MMP3, matrix metalloproteinase; ADAMTS, disintegrin and metalloproteinase with thrombospondin motifs.

present study, iNOS and COX-2 were found to be significantly upregulated following stimulation with IL-1 $\beta$. It has been previously reported that the production of iNOS and COX-2 serves an important role in the pathophysiology of OA (45). Several studies demonstrated that iNOS and COX-2 downregulation ameliorated the progression of OA $(46,47)$. The present findings showed that OrA significantly prevented the IL- $1 \beta$-induced expression of iNOS and COX-2. ECM is the main component of articular cartilage (48). Increased catabolism of ECM is considered to be a crucial factor in 
B A
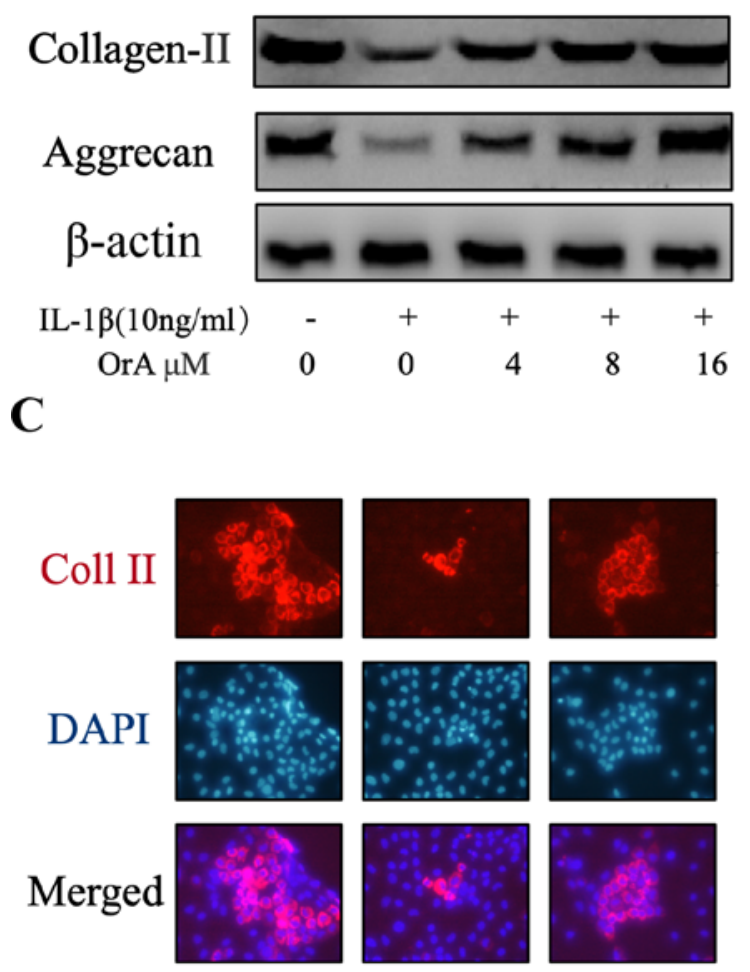

Control

IL-1 $\beta$

IL- $1 \beta+$ OrA
D
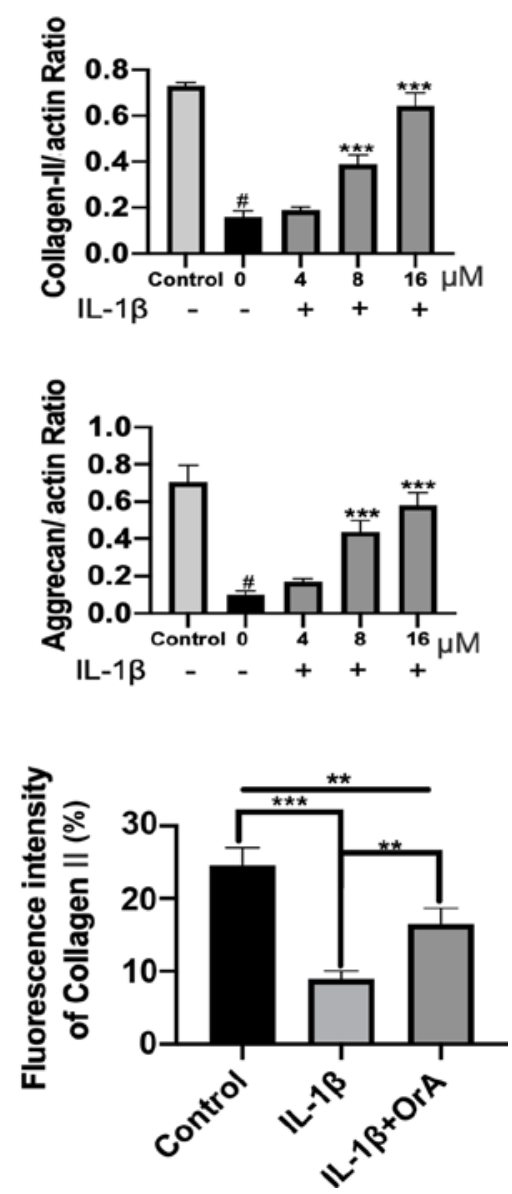

Figure 4. OrA reverses IL-1 $\beta$-induced degradation of ECM. Chondrocytes were treated with various concentrations of OrA (4, 8 and $16 \mu \mathrm{M})$ and stimulated with or without IL-1 $\beta$ (10 ng/ml) for $24 \mathrm{~h}$. (A) Protein expression levels of type II collagen and aggrecan were measured using western blot analysis. (B) Quantification of type II collagen and aggrecan expression from three experimental repeats. Data are expressed as the mean \pm standard deviation, ${ }^{\#} \mathrm{P}<0.01$ vs. untreated; ${ }^{* * *} \mathrm{P}<0.001$ vs. IL-1 $\beta$ only. (C) Immunofluorescence analysis of type II collage expression, (D) which was then quantified (original magnification, $\mathrm{x} 200)$. Each sample was analyzed thrice. Data are expressed as the mean \pm standard deviation from three experimental repeats. ${ }^{* *} \mathrm{P}<0.01$ and ${ }^{* * *} \mathrm{P}<0.001$. OrA, oroxylin A; ECM, extracellular matrix; IL-1 $\beta$, interleukin-1 $\beta$; Coll II, collagen II.

the progression of OA (49). Previous studies have provided evidence that the activation of MMPs, especially MMP3 and MMP13, promotes the degradation of $\operatorname{ECM}(12,13)$. In the present study, stimulation with IL-1 $\beta$ markedly upregulated the expression of MMP3 and MMP13, whilst pre-treatment with OrA prevented this effect. Aggrecan and type II collagen are the main components of ECM, such that downregulation of both of these molecules leads to cartilage degradation (50). In the present study, IL-1 $\beta$ significantly attenuated the expression of aggrecan and type II collagen, whilst pre-treatment with OrA prevented this effect. In addition, the ADAMTS enzymes, especially ADAMTS-4 and ADAMTS-5, are considered to be the primary aggrecanases with the ability to cleave aggrecans $(51,52)$. In the present study, treatment with OrA attenuated IL-1 $\beta$-induced expression of ADAMTS-5 and protected against IL-1 $\beta$-induced cartilage degradation.

The MAPK and PI3K/AKT signaling pathways serve a crucial role in the pathogenesis of OA $(53,54)$. Inhibition of IL-1 $\beta$-induced activation of ERK has been previously reported to attenuate the progress of OA (55). Accumulating evidence has suggested that the activation of ERK mediates the production of MMPs, thereby promoting cartilage degradation $(56,57)$. The present study demonstrated that treatment with OrA significantly inhibited the activation of ERK. Another previous study reported that inhibition of the PI3K/AKT signaling pathway relieved IL-1 $\beta$-induced inflammatory response in chondrocytes (58). In addition, it has been also reported that $\mathrm{PI} 3 \mathrm{~K} / \mathrm{AKT}$ signaling regulates the expression of aggrecan (59). Therefore, the present study investigated the effect of OrA on IL-1 $\beta$-mediated activation of the PI3K/AKT signaling pathway and confirmed that OrA could also inhibit the PI3K/AKT signaling pathway.

However, there are still several limitations in the current study. First, as the present research was based on murine chondrocytes, which were obtained from neonatal mice, the mice were not weighed or sexed upon purchase. Additionally, the current study lacks in vivo results, which should be assessed in future work.

Taken together, the results of the present study suggested that OrA exerted protective effects against IL-1 $\beta$-induced inflammatory response by inhibiting the activation of the ERK and PI3K/AKT signaling pathways. The current study indicated the therapeutic potential of OrA in osteoarthritis treatment and may therefore provide a novel candidate for OA therapy. 
A

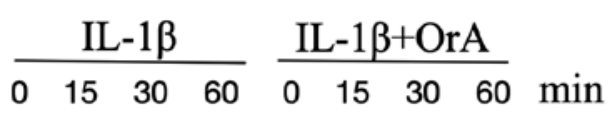

\section{P-p65}

p65

$\mathrm{I} \kappa \mathrm{B} \alpha$

$\beta$-actin
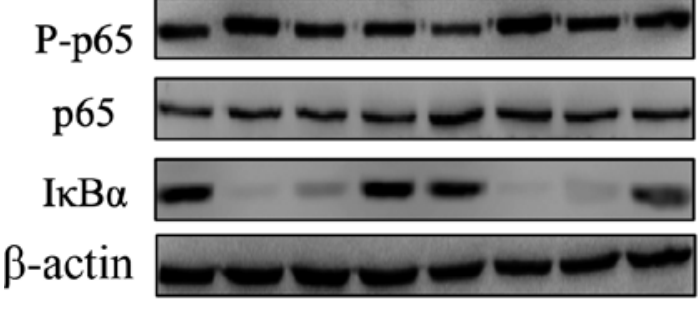

C

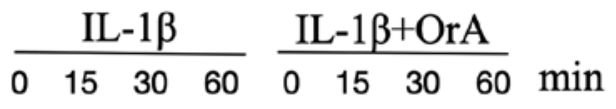

P-ERK

ERK

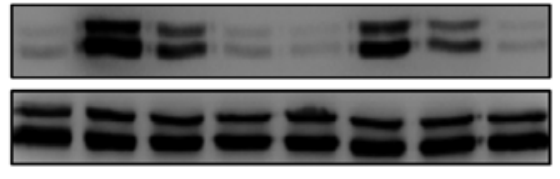

P-JNK

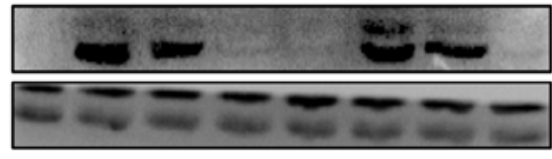

JNK

$$
\text { P-p38 }
$$

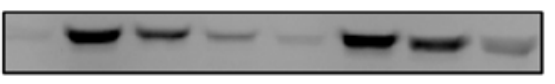

$$
\text { p38 }
$$

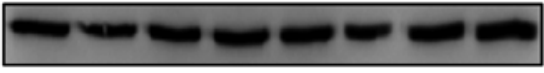

$\beta$-actin
B
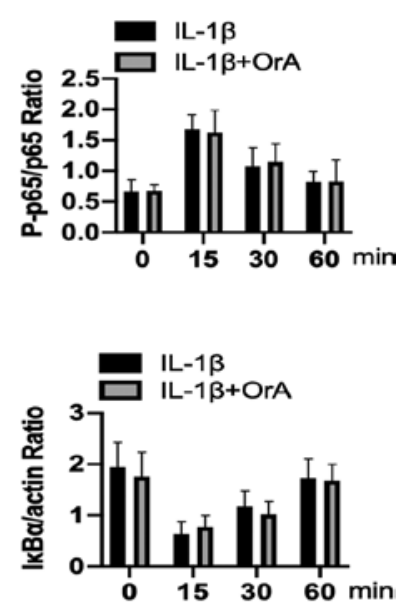

D
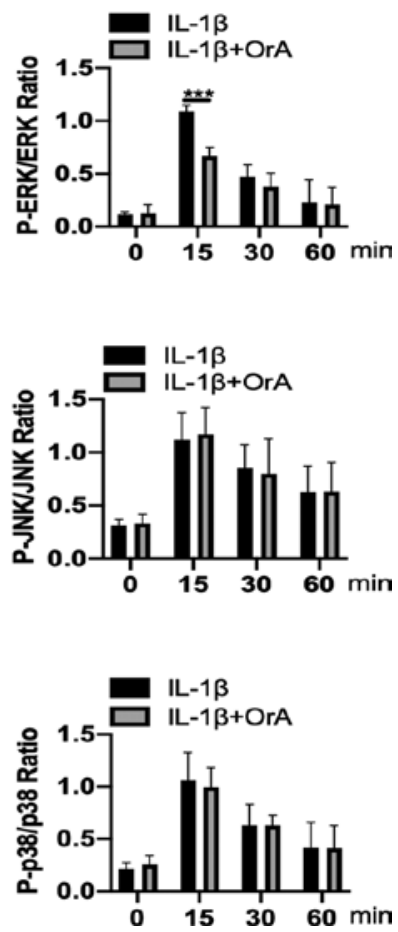

Figure 5. Effect of OrA on IL-1 $\beta$-mediated NF- $\kappa \mathrm{B}$ and MAPK activation. Chondrocytes were pre-treated with or without $16 \mu \mathrm{M}$ OrA for $2 \mathrm{~h}$, and then stimulated with or without IL-1 $\beta$ (10 ng/ml) for different time periods $(0,15,30$ and $60 \mathrm{~min})$. (A) The protein expression levels of p65 and IkB $\alpha$, in addition to 065 phosphorylation were determined by western blot analysis. (B) which was then quantified. (C) The protein expression levels of ERK, JNK, JNK and $\mathrm{p} 38$, in addition to their corresponding phosphorylation levels, were determined by western blot analysis and (D) quantified. Data are expressed as the mean \pm standard deviation from three experimental repeats. ${ }^{* * * *} \mathrm{P}<0.001$ vs. the IL-1 $\beta$ only group. OrA, oroxylin A; IL-1 $\beta$, interleukin-1 $\beta ;$ IкB $\alpha, N F-\kappa B$ inhibitor $\alpha$; p-, phosphorylated.

A

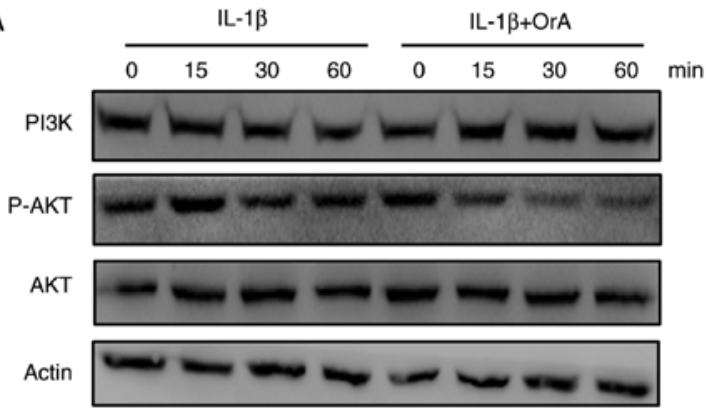

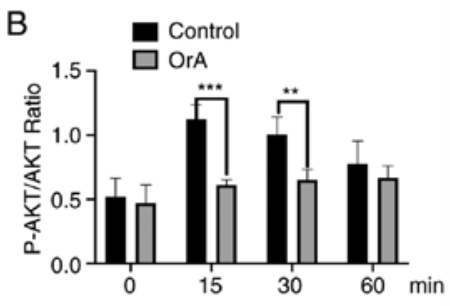

Figure 6. Effect of OrA on IL-1 $\beta$-mediated PI3K/AKT activation. (A) Protein expression levels of PI3K and AKT, in addition to AKT phosphorylation, were determined by western blot analysis and (B) quantified. Data are expressed as the mean \pm standard deviation from three experimental repeats. ${ }^{* *} \mathrm{P}<0.01$ and ${ }^{* * * *} \mathrm{P}<0.001$ vs. the IL-1 $\beta$ only group. OrA, oroxylin A; IL-1 $\beta$, interleukin-1 $\beta$. 


\section{Acknowledgements}

Not applicable.

\section{Funding}

The present study was supported by the Medical and Health Technology Project of Zhejiang Province (grant no. 2019PY073); Science and Technology Research on Public Welfare Project of Ningbo, Zhejiang Province (grant no. 2019C50050) and the Scientific Technology Project of Agriculture and Social Development of Yinzhou, Ningbo, Zhejiang Province (grant no. 20180137).

\section{Availability of data and materials}

The datasets used and/or analyzed during the present study are available from the corresponding author upon reasonable request.

\section{Authors' contributions}

YZ and QW conceived the study; YZ and JC conducted the experiments; $\mathrm{JH}$ wrote the manuscript and performed statistical analysis; QW and ML analyzed the results and created the figures. All authors read and approved the final manuscript.

\section{Ethics approval and consent to participate}

Animal experiments were conducted in accordance with the international ethical guidelines and the National Institutes of Health Guide for Care and Use of Laboratory Animals (NIH Pub No 85-23, revised 1996). The procedures were approved by the Ethics Committee of Ningbo No. 6 Hospital (Ningbo, China).

\section{Patient consent for publication}

Not applicable.

\section{Competing interests}

The authors declare that they have no competing interests.

\section{References}

1. Ji Q, Xu X, Kang L, Xu Y, Xiao J, Goodman SB, Zhu X, Li W, Liu J, Gao X, et al: Hematopoietic PBX-interacting protein mediates cartilage degeneration during the pathogenesis of osteoarthritis. Nat Commun 10: 313, 2019.

2. Ashford S and Williard J: Osteoarthritis: A review. Nurse Pract 39: 1-8, 2014.

3. Abramson SB: Inflammation in osteoarthritis. J Rheumatol Suppl 70: 70-76, 2004.

4. Chen YF, Jobanputra P, Barton P, Bryan S, Fry-Smith A, Harris G and Taylor RS: Cyclooxygenase-2 selective non-steroidal anti-inflammatory drugs (etodolac, meloxicam, celecoxib, rofecoxib, etoricoxib, valdecoxib and lumiracoxib) for osteoarthritis and rheumatoid arthritis: A systematic review and economic evaluation. Health Technol Assess 12: 1-278, 2008.

5. Lane NE: Pain management in osteoarthritis: The role of COX-2 inhibitors. J Rheumatol Suppl 49: 20-24, 1997.

6. Hungin APS and Kean WF: Nonsteroidal anti-inflammatory drugs: Overused or underused in osteoarthritis? Am J Med 110 (Suppl): S8-S11, 2001.

7. Felson DT: Risk factors for osteoarthritis: Understanding joint vulnerability. Clin Orthop Relat Res 427: 16-21, 2004.
8. Goldring MB and Otero M: Inflammation in osteoarthritis. Curr Opin Rheumatol 23: 471-478, 2011.

9. Mokuda S, Nakamichi R, Matsuzaki T, Ito Y, Sato T, Miyata K, Inui M, Olmer M, Sugiyama E, Lotz M and Asahara H: Wwp2 maintains cartilage homeostasis through regulation of Adamts5. Nat Commun 10: 2429, 2019.

10. Lee JH, Shehzad O, Ko SK, Kim YS and Kim HP: Matrix metalloproteinase-13 downregulation and potential cartilage protective action of the Korean Red Ginseng preparation. J Ginseng Res 39: 54-60, 2015.

11. Gao Y, Liu S, Huang J, Guo W, Chen J, Zhang L, Zhao B, Peng J, Wang A, Wang Y, et al: The ECM-cell interaction of cartilage extracellular matrix on chondrocytes. Biomed Res Int 2014: 648459, 2014

12. Wang M, Sampson ER, Jin H, Li J, Ke QH, Im HJ and Chen D: MMP13 is a critical target gene during the progression of osteoarthritis. Arthritis Res Ther 15: R5, 2013.

13. Kubota E, Imamura H, Kubota T, Shibata T and Murakami KI Interleukin-1 beta and stromelysin (MMP3) activity of synovial fluid as possible markers of osteoarthritis in the temporomandibular joint. J Oral Maxillofac Surg 55: 20-28, 1997.

14. Studer R, Jaffurs D, Stefanovic-Racic M, Robbins PD and Evans CH: Nitric oxide in osteoarthritis. Osteoarthritis Cartilage 7: 377-379, 1999.

15. Smith RL: Degradative enzymes in osteoarthritis. Front Biosci 4: D704-D712, 1999.

16. Tchetina EV, Squires G and Poole AR: Increased type II collagen degradation and very early focal cartilage degeneration is associated with upregulation of chondrocyte differentiation related genes in early human articular cartilage lesions. J Rheumatol 32: 876-886, 2005.

17. Vertel BM: The ins and outs of aggrecan. Trends Cell Biol 5: 458-464, 1995.

18. Ding J, Ghali O, Lencel P, Broux O, Chauveau C, Devedjian JC, Hardouin P and Magne D: TNF-alpha and IL-1beta inhibit RUNX2 and collagen expression but increase alkaline phosphatase activity and mineralization in human mesenchymal stem cells. Life Sci 84: 499-504, 2009.

19. Wang J, Markova D, Anderson DG, Zheng Z, Shapiro IM and Risbud MV: TNF- $\alpha$ and IL- $1 \beta$ promote a disintegrin-like and metalloprotease with thrombospondin type I motif-5-mediated aggrecan degradation through syndecan-4 in intervertebral disc. J Biol Chem 286: 39738-39749, 2011.

20. Zou M, Hu C, You Q, Zhang A, Wang X and Guo Q: Oroxylin $\mathrm{A}$ induces autophagy in human malignant glioma cells via the mTOR-STAT3-Notch signaling pathway. Mol Carcinog 54: 1363-1375, 2015

21. Yao J, Hu R, Sun J, Lin B, Zhao L, Sha Y, Zhu B, You QD, Yan T and Guo QL: Oroxylin a prevents inflammation-related tumor through down-regulation of inflammatory gene expression by inhibiting NF- $\kappa \mathrm{B}$ signaling. Mol Carcinog 53: 145-158, 2014.

22. Ye M, Wang Q, Zhang W, Li Z, Wang Y and Hu R: Oroxylin A exerts anti-inflammatory activity on lipopolysaccharide-induced mouse macrophage via Nrf2/ARE activation. Biochem Cell Biol 92: 337-348, 2014.

23. Li J, Tong D, Liu J, Chen F and Shen Y: Oroxylin A attenuates cigarette smoke-induced lung inflammation by activating Nrf2. Int Immunopharmacol 40: 524-529, 2016.

24. Han Q, Wang H, Xiao C, Fu BD and Du CT: Oroxylin A inhibits $\mathrm{H}_{2} \mathrm{O}_{2}$-induced oxidative stress in PC12 cells. Nat Prod Res 31: 1339-1342, 2017

25. Zou M, Lu N, Hu C, Liu W, Sun Y, Wang X, You Q, Gu C, Xi T and Guo Q: Beclin 1-mediated autophagy in hepatocellular carcinoma cells: Implication in anticancer efficiency of oroxylin A via inhibition of mTOR signaling. Cell Signal 24: 1722-1732, 2012.

26. Sun Y, Lu N, Ling Y, Gao Y, Chen Y, Wang L, Hu R, Qi Q, Liu W, Yang Y, et al: Oroxylin A suppresses invasion through down-regulating the expression of matrix metalloproteinase-2/9 in MDA-MB-435 human breast cancer cells. Eur J Pharmacol 603: 22-28, 2009.

27. Choi HW, Shin PG, Lee JH, Choi WS, Kang MJ, Kong WS, Kang MJ, Kong WS, Oh MJ, Seo YB and Kim GD: Anti-inflammatory effect of lovastatin is mediated via the modulation of NF- $\mathrm{NB}$ and inhibition of HDAC1 and the PI3K/Akt/mTOR pathway in RAW264. 7 macrophages. Int J Mol Med 41: 1103-119, 2018.

28. Chen YC, Yang LL and Lee TJF: Oroxylin A inhibition of lipopolysaccharide-induced iNOS and COX-2 gene expression via suppression of nuclear factor- $\kappa \mathrm{B}$ activation. Biochem Pharmacol 59: 1445-1457, 2000. 
29. Livak KJ and Schmittgen TD: Analysis of relative gene expression data using real-time quantitative PCR and the 2(-Delta Delta C(T)) method. Methods 25: 402-408, 2001.

30. Sherwin CM, Christiansen SB, Duncan IJ, Erhard HW, Lay DC, Mench JA, O'Connorg CE and Petherick CJ: Guidelines for the ethical use of animals in applied ethology studies. Appl Anim Behav Sci 81: 291-305, 2003.

31. Derrell CJ, Gebhart GF, Gonder JC, Keeling ME and Kohn DF: Special Report: The 1996 Guide for the care and use of laboratory animals. ILAR J 38: 41-48, 1997

32. Glasson SS, Askew R, Sheppard B, Carito B, Blanchet T, Ma HL, Flannery CR, Peluso D, Kanki K, Yang Z, et al: Deletion of active ADAMTS5 prevents cartilage degradation in a murine model of osteoarthritis. Nature 434: 644-648, 2005.

33. Poole AR, Kobayashi M, Yasuda T, Laverty S, Mwale F, Kojima T, Sakai T, Wahl C, El-Maadawy S, Webb G, et al: Type II collagen degradation and its regulation in articular cartilage in osteoarthritis. Ann Rheum Dis 61 (Suppl 2): ii78-ii81, 2002.

34. Song RH, Tortorella MD, Malfait AM, Alston JT, Yang Z, Arner EC and Griggs DW: Aggrecan degradation in human articular cartilage explants is mediated by both ADAMTS- 4 and ADAMTS-5. Arthritis Rheum 56: 575-585, 2014.

35. Glyn-Jones S, Palmer AJR, Agricola R, Price AJ, Vincent TL, Weinans H and Carr AJ: Osteoarthritis. Lancet 386: 376-387, 2015.

36. Zhang Y and Jordan JM: Epidemiology of osteoarthritis. Clin Geriatr Med 26: 355-369, 2010.

37. Sarzi-Puttini P, Cimmino MA, Scarpa R, Caporali R, Parazzini F, Zaninelli A, Atzeni F and Canesi B: Osteoarthritis: An overview of the disease and its treatment strategies. Semin Arthritis Rheum 35: 1-10, 2005.

38. Giercksky KE, Huseby G and Rugstad HE: Epidemiology of NSAID-related gastrointestinal side effects. Scand J Gastroenterol Suppl 163: 3-8, 1989.

39. Lichtenberger LM, Zhou Y, Dial EJ and Raphael RM: NSAID injury to the gastrointestinal tract: Evidence that NSAIDs interact with phospholipids to weaken the hydrophobic surface barrier and induce the formation of unstable pores in membranes. J Pharm Pharmacol 58: 1421-1428, 2010.

40. Song X, Chen Y, Sun Y, Lin B, Qin Y, Hui H, Li Z, You Q, Lu N and Guo Q: Oroxylin A, a classical natural product, shows a novel inhibitory effect on angiogenesis induced by lipopolysaccharide. Pharmacol Rep 64: 1189-1199, 2012.

41. Sun X, Chang X, Wang Y, Xu B and Cao X: Oroxylin A suppresses the cell proliferation, migration, and EMT via NF- $\kappa B$ signaling pathway in human breast cancer cells. Biomed Res Int 2019: 9241769, 2019

42. Roos EM and Arden NK: Strategies for the prevention of knee osteoarthritis. Nat Rev Rheumatol 12: 92, 2016.

43. Luo Y, Sinkeviciute D, He Y, Karsdal M, Henrotin Y, Mobasheri A, Önnerfjord P and Bay-Jensen A: The minor collagens in articular cartilage. Protein Cell 8: 560-572, 2017.

44. Bonassar LJ, Sandy JD, Lark MW, Plaas AHK, Frank EH and Grodzinsky AJ: Inhibition of cartilage degradation and changes in physical properties induced by IL-1 $\beta$ and retinoic acid using matrix metalloproteinase inhibitors. Arch Biochem Biophys 344: 404-412, 1997

45. Needleman P and Manning PT: Interactions between the inducible cyclooxygenase (COX-2) and nitric oxide synthase (iNOS) pathways: Implications for therapeutic intervention in osteoarthritis. Osteoarthritis Cartilage 7: 367-370, 1999.
46. More AS, Kumari RR, Gupta G, Lingaraju MC, Balaganur V, Pathak NN, Kumar D, Kumar D, Sharma AK and Tandan SK: Effect of iNOS inhibitor S-methylisothiourea in monosodium iodoacetate-induced osteoathritic pain: Implication for osteoarthritis therapy. Pharmacol Biochem Behav 103: 764-772, 2013.

47. Watson DJ, Harper SE, Zhao PL, Quan H, Bolognese JA and Simon TJ: Gastrointestinal tolerability of the selective cyclooxygenase-2 (COX-2) inhibitor rofecoxib compared with nonselective COX-1 and COX-2 inhibitors in osteoarthritis. Arch Intern Med 160: 2998-3003, 2000.

48. Wong $\mathrm{M}$ and Carter DR: Articular cartilage functional histomorphology and mechanobiology: A research perspective. Bone 33: $1-13,2003$

49. Rahmati M, Nalesso G, Mobasheri A and Mozafari M: Aging and osteoarthritis: Central role of the extracellular matrix. Ageing Res Rev 40: 20-30, 2017.

50. Pattoli MA, MacMaster JF, Gregor KR and Burke JR: Collagen and aggrecan degradation is blocked in interleukin-1-treated cartilage explants by an inhibitor of IкB kinase through suppression of metalloproteinase expression. J Pharmacol Exp Ther 315: 382-388, 2005.

51. Stanton H, Rogerson FM, East CJ, Golub SB, Lawlor KE, Meeker CT, Little CB, Last K, Farmer PJ, Campbell IK, et al: ADAMTS5 is the major aggrecanase in mouse cartilage in vivo and in vitro. Nature 434: 648-652, 2005.

52. Song RH, Tortorella MD, Malfait AM, Alston JT, Yang Z, Arner EC and Griggs DW: Aggrecan degradation in human articular cartilage explants is mediated by both ADAMTS-4 and ADAMTS-5. Arthritis Rheum 56: 575-585, 2007.

53. Shi J, Zhang C, Yi Z and Lan C: Explore the variation of MMP3, JNK, p38 MAPKs, and autophagy at the early stage of osteoarthritis. IUBMB Life 68: 293-302, 2016.

54. Chow YY and Chin KY: The role of inflammation in the pathogenesis of osteoarthritis. Mediators Inflamm 2020: 8293921, 2020.

55. Gao SC, Yin HB, Liu HX and Sui YH: Research progress on MAPK signal pathway in the pathogenesis of osteoarthritis. Zhongguo Gu Shang 27: 441-444, 2014 (In Chinese).

56. Liu Z, Cai H, Zheng X, Zhang B and Xia C: The involvement of mutual inhibition of ERK and mTOR in PLC $\gamma 1$-mediated MMP-13 expression in human osteoarthritis chondrocytes. Int J Mol Sci 16: 17857-17869, 2015.

57. Hashizume $M$ and Mihara M: High molecular weight hyaluronic acid inhibits IL-6-induced MMP production from human chondrocytes by up-regulating the ERK inhibitor, MKP-1. Biochem Biophys Res Commun 403: 184-189, 2010.

58. Xue JF, Shi ZM,Zou J and Li XL: Inhibition of PI3K/AKT/mTOR signaling pathway promotes autophagy of articular chondrocytes and attenuates inflammatory response in rats with osteoarthritis. Biomed Pharmacother 89: 1252-1261, 2017.

59. Cheng CC, Uchiyama Y, Hiyama A, Gajghate S, Shapiro IM and Risbud MV: PI3K/AKT regulates aggrecan gene expression by modulating Sox 9 expression and activity in nucleus pulposus cells of the intervertebral disc. J Cell Physiol 221: 668-676, 2009.

(i) $($ This work is licensed under a Creative Commons Attribution-NonCommercial-NoDerivatives 4.0 International (CC BY-NC-ND 4.0) License. 